\title{
Mathematical notation
}

A vector is usually denoted by a lower case italic letter such as $x$ or $y$, and sometimes is represented with an arrow on top of the letter such as $\vec{x}$ or $\vec{y}$. Sometimes a vector is described by enumeration of its elements; in these cases subscripts are used to denote individual elements of a vector and superscripts to denote a specific one: $x=\left(x_{1}, \ldots, x_{n}\right)$ means a generic $\mathrm{n}$ dimensional vector and $x^{0}=\left(x_{1}^{0}, \ldots, x_{n}^{0}\right)$ a specific $\mathrm{n}$-dimensional vector. As it is usual, $x>>$ $y$ means $x_{i}>y_{i}(\mathrm{i}=1, \ldots, \mathrm{n})$ and $x>y$ means $x_{i} \geq y_{i}$ for all $\mathrm{i}$ and, for at least one $\mathrm{i}, x_{i}>y_{i}$.

A set is denoted by a capital italic letter such as $X$ or $Y$. If a set is defined by some property of its members, it is written with brackets which contain in the first place the typical element followed by a vertical line and the property: $X=(x / x>>0)$ is the set of vectors $x$ with positive elements. In particular, $R$ is the set of real numbers, $R_{+}$the set of non-negative real numbers, $R_{++}$the set of positive real numbers and a superscript denotes the dimension of the set. $\mathrm{R}_{+}^{n}$ is the set of $\mathrm{n}$-dimensional vectors whose elements are all real non-negative numbers.

Matrices are denoted by capital italic letters such as $A$ or $B$, or by squared brackets surrounding their typical element $\left[a_{i j}\right]$ or $\left[b_{i j}\right]$. When necessary, $A(q x m)$ indicates that matrix $A$ has $q$ rows and $m$ columns (is of order $q x m$ ).

In equations systems expressed in matricial form it is supposed that dimensions of matrices and vectors are the right ones, therefore we do not use transposition symbols. For example, in the system $y=A x+u$, with $A(n x n)$, all the three vectors must have $n$ rows and 1 column but they are represented ini the text as $y=\left(y_{1}, \ldots, y_{n}\right), x=\left(x_{1}, \ldots, x_{n}\right)$ and $u=\left(u_{1}, \ldots, u_{n}\right)$. The only exceptions are when expressing a quadratic form such as $x A x^{\prime}$ or a matricial product such as $\left(X^{\prime} X\right)^{-1}$.

The remaining notation is the standard use for mathematics, and when more specific notation is used it is explained in the text. 